\title{
Impacto Económico de la Vacuna contra el Neumococo en Pacientes Oncológicos
}

\section{Economic impact of neumococcal vaccine on oncological patients}

\author{
Liliana A. Chicaíza-Becerraํㅜ, Mario García-Molina, Mónica Ballesteros², Oscar \\ Gamboa $^{2}$, Jorge Díaz ${ }^{1}$, Ricardo Vega ${ }^{1}$ y Fernando de la Hoz ${ }^{1}$
}

1. Facultad de Ciencias Económicas, Facultad de Ciencias, Facultad de Medicina. Universidad Nacional de Colombia. lachicaizab@unal.edu.co,mgarciamo@unal.edu.co,ravegaro@unal.edu.co, jadiazr@unal.edu.co,fpdelahozr@unal.edu.co

2. Grupo de Investigación Clínica, Instituto Colombiano de Cancerología.

mpballesteross@unal.edu.co,ogamboag@unal.edu.co

Recibido 12 Diciembre 2006/Enviado para Modificación 14 Julio 2007/Aceptado 25 Agosto 2007

\section{RESUMEN}

Objetivos Evaluar económicamente la vacuna contra $S$ pneumoniae en pacientes oncológicos para Colombia.

Métodos Dado que la efectividad de la vacuna en este tipo de pacientes no está comprobada, se calculó el costo de los primeros seis años de un programa de vacunación para pacientes oncológicos y se estimó el costo de oportunidad con alternativas de detección temprana de cáncer.

Resultados Un programa de vacunación de pacientes oncológicos contra $S$ pneumoniae costaría en sus primeros seis años cerca de treinta y un mil millones de pesos constantes de 2006. Con estos recursos se podrían realizar aproximadamente 170000 colonoscopias o pruebas de detección temprana de VPH, 500000 mamografías o más de 3800000 citologías.

Conclusiones Dada la escasez de recursos, es preferible económicamente destinar estas cifras a adelantar programas cuya efectividad esté comprobada, ya sea para detección temprana de cáncer o para vacunación de niños sanos.

Palabras Clave: Infecciones neumocócicas, economía, costos y análisis de costo, vacunación, cáncer (fuente: DeCS, BIREME).

\section{ABSTRACT}

Objective An economic evaluation of an anti-S pneumoniae vaccine for oncological patients in Colombia.

Methods As there is no evidence of vaccine effectiveness for this kind of patient, the cost of a vaccination programme for oncological patients was calculated during its first six years and the opportunity cost was estimated for early cancer detection alternatives.

Results An anti-S. pneumoniae vaccination programme for oncological patients would cost around $\$ 31000000000$ (Colombian pesos in 2006, i.e. nearly US\$12 
$400000)$ during its first years. Alternative programs could be developed with this amount, such as 170000 colonoscopies or early HPV detection, 500000 mammographies, or more than 3800000 cytologies.

Conclusions Given the scarcity of resources, it would be better (from an economic point of view) to devote this amount to programmes whose effectiveness has been proven, such as early cancer detection or vaccinating healthy children.

Key Words: Pneumococcal infection, economics, costs and cost analysis, vaccination, neoplasm (source: $M e S H, N L M$ ).

$\mathrm{E}$

I Streptococcus pneumoniae (pneumococcus) es un patógeno bacteriano que afecta niños y adultos alrededor del mundo. El microorganismo coloniza el tracto respiratorio superior y puede causar enfermedades invasivas diseminadas, así como enfermedades de los tractos respiratorio inferior y superior (1).

Se estima que cada año en Estados Unidos la enfermedad neumocócica provoca aproximadamente 40000 muertes; esta cifra es mayor a la correspondiente a cualquier otra enfermedad bacteriana prevenible por vacunación (2). La incidencia anual de bacteriemia por neumococo se ha estimado en 15-30 casos por 100000 personas (3). No esta documentado el incremento de la incidencia de la enfermedad pneumocócica en pacientes con enfermedades crónicas (4).

Los niños menores de 2 años, los adultos mayores de 65 años y las personas con ciertas condiciones médicas tales como enfermedades cardiovasculares, pulmonares y hepáticas crónicas tienen un riesgo incrementado de infección pneumocócica, así mismo las personas con respuestas disminuidas a antígenos de polisacáridos o concentraciones disminuidas de anticuerpos como resultado de condiciones inmunosupresivas, transplantes, ciertas terapias y falla renal crónica.

En Colombia, según las estadísticas de Vigilancia en Salud Publica del Instituto Nacional de Salud entre 1994 y 2005, el número de aislamientos invasores de este microorganismo, en menores de 5 años fue 1315 , entre niños de 6 a 14 años fue de 237 y, en mayores de 14 años, 756 (5).

Durante 2004 se registraron en el Instituto Nacional de Cancerología 15 muertes atribuibles a enfermedades respiratorias, para una tasa de 11,74 defunciones atribuibles a enfermedades respiratorias por cada 1000 muertes 
de pacientes oncológicos. La causa básica de muerte relacionada en el certificado de defunción fue en doce casos neumonía, en dos enfermedades crónicas de las vías respiratorias inferiores y en un caso otras enfermedades del sistema respiratorio. El rango de edad en un 86,6 \% de los casos eran pacientes de 65 o más años de edad. No se relacionó esta información con los agentes microbianos causales (6). En pacientes con cáncer, se desconocen la frecuencia de presentación y la tasa de mortalidad a nivel nacional por enfermedades respiratorias.

Una de las maneras efectivas de modificar las tasas de morbilidad y de mortalidad producidas por algunas enfermedades infecciosas es el uso de la vacunación, con el fin de estimular al sistema inmune del huésped para que el mismo sea capaz de producir anticuerpos que le brinden protección prolongada en el tiempo al entrar en contacto con un antígeno previamente conocido.

En la actualidad se encuentran disponibles dos vacunas para Streptococcus pneumoniae: una vacuna de 23 polisacáridos y una conjugada o heptavalente. Después de la vacunación, la respuesta de anticuerpos a un antígeno especifico, se indica por el aumento de dos veces o mas en la cantidad de anticuerpos, que desarrollan en las 2-3 semanas siguientes el $80 \%$ de adultos saludables (1); sin embargo la respuesta no es consistente para todos los 23 serotipos de la vacuna (1).

En pacientes inmunocomprometidos, la respuesta de anticuerpos a la vacunación contra $S$. pneumoniae está frecuentemente disminuida o ausente. En pacientes con leucemia, linfoma y mieloma múltiple, la respuesta de anticuerpos a la vacunación contra neumococo es sustancialmente más baja que la respuesta entre pacientes inmunocompetentes (7). En pacientes con enfermedad de Hodgkin la respuesta de anticuerpos a la vacunación es mas alta, si esta es administrada antes de la esplenectomía, radiación o quimioterapia, sin embargo, durante la quimioterapia, los anticuerpos preexistentes pueden decrecer y la respuesta a la vacunación estaría disminuida (1).

Para personas inmunocompetentes se sabe que la vacuna contra $S$. pneumoniae, disminuye morbilidad y mortalidad secundarias a infecciones del tracto respiratorio. Un reciente estudio que evalúa el resultado de 259627 personas de 65 años o más a quienes se les suministró la vacuna de la influenza y neumococo, mostró que la incidencia de tratamiento hospitalario para la influenza, de la neumonía por influenza, de la neumonía por neumococo secundaria a la infección por el virus de la Influenza y de enfermedad 
neumocócica invasiva fue significativamente menor en el grupo vacunado, comparado con el grupo de no vacunados, y la mortalidad total fue $57 \%$ menor en el grupo de individuos vacunados (8).

Aunque la eficacia de la vacuna está comprobada para pacientes inmunocompetentes, no se deduce automáticamente la eficacia para pacientes oncológicos que es el caso en cuestión. Más aún, la eficacia de la vacuna debería referirse al desenlace clínico y no simplemente a conteo de anticuerpos.

El Grupo de Investigación Clínica en Cáncer del Instituto Nacional de Cancerología realizó una revisión sistemática de la literatura cuyo principal objetivo fue determinar la eficacia de la vacunación contra $S$. pneumoniae para disminuir mortalidad, evitar neumonía, prevenir enfermedades invasivas y generar anticuerpos protectores en pacientes oncológicos (7). Se excluyeron de la búsqueda estudios de pacientes con VIH-SIDA, post transplante de médula ósea y esplenectomisados. No hubo restricciones de edad, sexo, país o idioma. Una vez identificados los términos, se buscó en Embase, MEDLINE, Lilacs, CINAHL y Cochrane. En total se encontraron 337 artículos, luego de una selección realizada según los criterios de elegibilidad por expertos temáticos y metodológicos del Instituto Nacional de Cancerología se escogieron 55 artículos. Al final se identificó que sólo tres artículos cumplían a cabalidad los criterios de elegibilidad por lo que fueron revisados de forma pareada y calificados según las plantillas de evaluación de calidad de SIGN (Scottish Intercollegiate Guidelines Network). Se encontró sólo un artículo con desenlace clínico para vacunación en pacientes oncológicos; este artículo (9) no muestra evidencia de eficacia de la vacuna de 23 valencias en pacientes oncológicos.

Cabe anotar que existen trabajos anteriores sobre vacunas, tal como la de 14 valencias (10). Estos resultados, no obstante, no son comparables en términos de evidencia porque la vacuna no es la misma y en ese momento la esplenectomía era una práctica usual.

A pesar de la falta de evidencia, la OMS (1) recomendó la vacunación contra S. pneumoniae para diversos grupos, dentro de los cuáles están incluidos los pacientes oncológicos. Otros entes regulatorios también han recomendado la vacunación $(11,12)$.

Esta recomendación se basa en que, si bien no hay evidencia de un beneficio absoluto de la vacunación en pacientes oncológicos, se plantea como una intervención cuyo riesgo beneficio es alta, ya que no se le hace ningún daño a 
los pacientes y se pueden obtener beneficios no contabilizados en ensayos clínicos.

Las recomendaciones mencionadas parten de criterios médicos, sin tomar en cuenta elementos económicos. Si bien hay antecedentes de evaluación de costo de vacunas contra el neumococo (10), no se han realizado evaluaciones para las vacunas actualmente en uso. Se requiere por lo tanto realizar una evaluación económica que mida el impacto de introducir la vacuna como parte del tratamiento para pacientes con cáncer en Colombia.

\section{METODOLOGÍA}

Con la evaluación económica se busca identificar la mejor forma de asignar los recursos teniendo en cuenta que ellos son escasos y que una vez se decide asignar recursos a una alternativa se pierde la oportunidad de asignarlos a otra. Dado que siempre que se escoge se renuncia a alguna alternativa, con la evaluación económica se busca que la asignación escogida produzca el mayor beneficio. En este sentido el costo de oportunidad identifica el valor de la alternativa que no se realizó. En este caso debido a que no se encontró evidencia se procede a identificar el valor que tendría implementar un programa de vacunación contra el neumococo en pacientes oncológicos en Colombia y lo que se podría hacer si se aplicaran estos mismos recursos en alternativas de detección temprana de cáncer con efectividad comprobada. Es decir, mediante el método de costo de oportunidad, se identificaría lo que podría realizarse en usos alternativos (13-15).

Para ello se estima el costo durante los primeros seis años de vacunar contra el neumococo a todos los pacientes oncológicos que hay en el país, tal como recomienda la Organización Mundial de la Salud. La metodología para la estimación del número de vacunas es la siguiente. Como el primer año se vacunaría a todos los enfermos de cáncer, el número de vacunas corresponde a la prevalencia estimada para el primer año. Durante los siguientes cuatro años, se vacunarían los nuevos casos. Para el sexto año, se vacunan a los nuevos casos de ese año más los enfermos vacunados el primer año que hayan sobrevivido, puesto que la vacuna debe administrarse nuevamente a los cinco años. Las cifras se refieren siempre a todos los tipos de cáncer excepto piel.

En ausencia de datos confiables, se asumió una prevalencia en el país para el año inicial de 130000 casos y se realizó un análisis de sensibilidad. 
Para el periodo 1995-1999 se estimaron en promedio por año 61641 casos nuevos de cáncer en Colombia, 28137 en hombres y 33504 en mujeres (16). Para el período 2002, Globocan estima una incidencia de 70750 nuevos casos. Se tomó la estimación de Globocan para todo el período.

Para la supervivencia a 5 años, se tomaron los principales tipos de cáncer según supervivencia por sexo y se asumió que la supervivencia a 5 años para los demás era de cero. Este estimativo subestima el número de personas a vacunar después de cinco años y por lo tanto el costo total.

Los tipos de cáncer de mayor incidencia para hombres son Próstata, Estómago, Pulmón y Colon/recto; para mujeres son Cuello Uterino, Mama, Estómago y Colón/recto. Para el cáncer de pulmón se asumió una supervivencia a 5 años nula y en su lugar se introdujo el linfoma Hodgkin con una supervivencia de $50 \%$; así mismo, se tomó en cuenta la leucemia con supervivencia del 10 \%. Para los anteriores tipos de cáncer se asumieron las supervivencias a 5 años que aparecen en la Tabla 1, las cuales fueron construidas a partir de los datos internacionales de la IARC (17).

Tabla 1. Supuestos de supervivencia a 5 años Colombia- Ambos sexos todas las edades

\begin{tabular}{lc}
\hline \multicolumn{1}{c}{ Tipo de Cáncer } & Supervivencia a 5 años (\%) \\
\hline Estómago & 10 \\
Colon y recto & 35 \\
Mama & 50 \\
Cuello uterino & 50 \\
Próstata & 50 \\
Linfoma Hodgkin & 80 \\
Leucemia & 10 \\
Otros & 0 \\
\hline
\end{tabular}

En ausencia de datos de prevalencia, se asumió que la distribución de los tipos de cáncer mencionados era igual a la incidencia para Colombia estimada por Globocan (18). Esto equivale a suponer una tasa promedio de supervivencia a 5 años de 17,6 \% para los pacientes existentes el primer año.

A partir de la información anterior, se calculó el costo de los seis años iniciales del programa, tomando como referencia el precio de mercado de la vacuna contra el neumococo, que se fijó en \$60 000 . 
El costo de oportunidad corresponde a lo que se puede hacer si la totalidad de los recursos que consume el programa de vacunación se destinan a otros programas. Como alternativas se escogieron programas que si tuvieran efectividad demostrada para la detección temprana de algunos tipos de cáncer, a saber detección temprana mediante citología, colposcopia, pruebas de VPH.

Otros métodos de evaluación económica, tales como costo efectividad, costo utilidad o costo beneficio, no eran aplicables aquí, dado que no hay evidencia de la efectividad de la vacuna. En otras palabras, el costo por unidad de resultado sería infinito.

\section{RESULTADOS}

A partir de los supuestos de la sección anterior se calculó el número de vacunas y se estimó su costo, suponiendo que el valor unitario de la vacuna fuera de $\$ 60$ 000. Los resultados aparecen en la Tabla 2.

Tabla 2. Vacunas estimadas y costos a 6 años

\begin{tabular}{|c|c|c|c|c|c|c|c|}
\hline Total & Año 1 & Año 2 & Año 3 & Año 4 & Año 5 & Año 6 & $\begin{array}{c}\text { Acumulado } \\
\text { a } 6 \text { años }\end{array}$ \\
\hline Incidencia & & 70750 & 70750 & 70750 & 70750 & 70750 & \\
\hline Prevalencia & 130000 & & & & & & \\
\hline Vivos 5 años & & & & & & 23200 & \\
\hline Vacunas & 130000 & 70750 & 70750 & 70750 & 70750 & 93950 & 506950 \\
\hline Precio & 60000 & 60000 & 60000 & 60000 & 60000 & 60000 & 60000 \\
\hline $\begin{array}{l}\text { Valor en } \\
\text { millones de } \\
\text { pesos- } 2006\end{array}$ & 7800 & 4245 & 4245 & 4245 & 4245 & 5637 & 30417 \\
\hline
\end{tabular}

La última fila de la Tabla 3 muestra los costos estimados a precios de 2006. Para tener una idea más clara de lo que estas cifras significan, se calculó el costo de oportunidad en opciones de prevención temprana de cáncer. La Tabla 3 muestra lo que se podría hacer con esos mismos montos.

Puesto que no existen datos confiables de prevalencia, se realizó una prueba de sensibilidad fijando este dato en 140000 en el primer año. El número y valor de las vacunas serían los de la Tabla 4 y los costos de oportunidad aparecen en la Tabla 5. Como se puede apreciar, los cambios no son muy grandes, son menores al 2,5\%. 
Tabla 3. Costo de Oportunidad

\begin{tabular}{lrrrrrrr}
\hline \multicolumn{1}{c}{ Total } & \multicolumn{1}{c}{ Año 1 } & \multicolumn{1}{c}{ Año 2 } & \multicolumn{1}{c}{ Año 3 } & \multicolumn{1}{c}{ Año 4 } & \multicolumn{1}{c}{ Año 5 } & Año 6 & $\begin{array}{c}\text { Acumulado } \\
\text { a 6 años }\end{array}$ \\
\hline S colonoscopia & 180000 & 180000 & 180000 & 180000 & 180000 & 180000 & \\
\# colonoscopia & 43333 & 23583 & 23583 & 23583 & 23583 & 31317 & 168983 \\
\$ mamografias & 60000 & 60000 & 60000 & 60000 & 60000 & 60000 & \\
\# mamografias & 130000 & 70750 & 70750 & 70750 & 70750 & 93950 & 506950 \\
\$ citología & 8000 & 8000 & 8000 & 8000 & 8000 & 8000 & \\
\# citologías & 975000 & 530625 & 530625 & 530625 & 530625 & 704626 & 3802126 \\
\$ Detección VPH & 180000 & 180000 & 180000 & 180000 & 180000 & 180000 & \\
\# Detección VPH & 43333 & 23583 & 23583 & 23583 & 23583 & 31317 & 168983 \\
\hline
\end{tabular}

Tabla 4. Costos alternativos

\begin{tabular}{lccccccc}
\hline Total & Año 1 & Año 2 & Año 3 & Año 4 & Año 5 & Año 6 & $\begin{array}{c}\text { Acumulado } \\
\text { a 6 años }\end{array}$ \\
\hline Incidencia & & 70750 & 70750 & 70750 & 70750 & 70750 & \\
$\begin{array}{l}\text { Prevalencia } \\
\text { Vivos 5 años }\end{array}$ & 140000 & & & & & & \\
Vacunas & 140000 & 70750 & 70750 & 70750 & 70750 & 95735 & 518735 \\
$\begin{array}{l}\text { Precio } \\
\text { Valor en \$000 }\end{array}$ & 60000 & 60000 & 60000 & 60000 & 60000 & 60000 & 60000 \\
000 de 2006 & 8400 & 4245 & 4245 & 4245 & 4245 & 5744 & 31124 \\
\hline
\end{tabular}

Tabla 5. Costo de oportunidad alternativos

\begin{tabular}{lrrrrrrr}
\hline Total & \multicolumn{1}{c}{ Año 1 } & \multicolumn{1}{c}{ Año 2 } & \multicolumn{1}{c}{ Año 3 } & \multicolumn{1}{c}{ Año 4 } & \multicolumn{1}{c}{ Año 5 } & \multicolumn{1}{c}{ Año 6 } & $\begin{array}{c}\text { Acumulado } \\
\text { a 6 años }\end{array}$ \\
\hline \$ colonoscopia & 180000 & 180000 & 180000 & 180000 & 180000 & 180000 & \\
\# colonoscopia & 46667 & 23583 & 23583 & 23583 & 23583 & 31912 & 172912 \\
\$ mamografias & 60000 & 60000 & 60000 & 60000 & 60000 & 60000 & \\
\# mamografias & 140000 & 70750 & 70750 & 70750 & 70750 & 95735 & 518735 \\
\$ citología & 8000 & 8000 & 8000 & 8000 & 8000 & 8000 & \\
\# citologías & 1050000 & 530625 & 530625 & 530625 & 530625 & 718011 & 3890511 \\
\$ Detección VPH & 180000 & 180000 & 180000 & 180000 & 180000 & 180000 & \\
\# Detección VPH & 46667 & 23583 & 23583 & 23583 & 23583 & 31912 & 172912 \\
\hline
\end{tabular}




\section{DISCUSIÓN}

Este artículo realiza una evaluación económica para la política propuesta por la OMS de vacunar a los pacientes oncológicos contra el neumococo. Aunque se han realizado evaluaciones económicas para otros casos (10), sus resultados no son comparables para la situación actual (vacuna contra 23 serotipos en ausencia de esplenectomía). Puesto que no hay evidencia con desenlace clínico fuerte, se optó por realizar un análisis de costo de oportunidad para identificar los usos alternativos de esos recursos. En ausencia de evidencia de efectividad de la vacuna cualquier asignación orientada a su aplicación en pacientes oncológicos podría considerarse ineficiente independientemente del costo de la vacuna y de cualquier tipo de focalización que se realice al interior del grupo de pacientes oncológicos.

Haciendo un cálculo conservador, la vacunación contra el neumococo en pacientes oncológicos significaría gastar en un procedimiento que no presenta evidencia clara de éxito más de treinta mil millones de pesos en seis años, cifra con la cual se podrían realizar setenta y seis mil colonoscopias, medio millón de mamografías o más de un millón de citologías en el período. Desde el punto de vista económico, dado que los recursos son escasos, sería más conveniente destinar estos recursos a procedimientos que tengan una efectividad comprobada. Recursos destinados a la detección temprana del cáncer o a la vacunación de niños sanos pueden ser, desde este punto de vista, más adecuados a las necesidades y capacidades del país.

Desde una lógica estrictamente económica, la recomendación de la OMS a favor de la vacuna porque no hace ningún daño, sólo sería aplicable en el caso de recursos ilimitados o, al menos, de países desarrollados. En un país en desarrollo destinar recursos a procedimientos cuya efectividad no está comprobada significa dejar de asignarlos a otras actividades que sí son efectivas. En otras palabras, en una situación de escasez de recursos como la colombiana, no basta con no hacer daño, es necesario que las medidas sean efectivas.

Cabe anotar que en este artículo no se está diciendo que las alternativas sean más costo efectivas que la vacunación, sino que, ante la falta de evidencia de efectividad de la vacunación para pacientes oncológicos, cualquier alternativa con efectividad demostrada sería preferible. El cálculo de costo de oportunidad sirve para tener una idea del orden de magnitud de lo que se estaría perdiendo al asignar recursos a un programa de vacunación como el estudiado 


\section{REFERENCIAS}

1. Centers for Disease Control and Prevention. [Internet]. Prevention of Pneumococcal Disease. Morbidity and Mortality Weekly Report. Abril 4. 1997, 46(RR-8) Disponible en: http://www.cdc.gov/ Consultado Junio de 2005.

2. Cetron MS, Jernigan DB, Breiman RF, DRSPWorking Group. Action plan for drugresistant Streptococcus pneumoniae. Emerg Infect Dis. 1995; Apr-Jun, 1(1): 6465.

3. Robinson KA, Baughman W, Rothrock G Barret NL, Pass M, Lexau C, et al. Epidemiology of invasive Streptococcus pneumoniae infections in the United States, 19951998. JAMA2001; 285: 1729-35.

4. Sanford J. Pneumococcal Vaccine: Clinical Efficacy and Effectiveness. Ann Intern Med, 1982; 96: 208-220.

5. Instituto Nacional de Salud. [internet]. Streptococcus pneumoniae (aislamientos invasores). Disponible en: http://www.ins.gov.co/pdf_investiga/ influenza_y_otros_virusresp_2006_18.pdf Consultado Noviembre de 2006.

6. Piñeros M, FerlayJ, MurilloR. Incidencia estimada y mortalidad por cáncer en Colombia, 1995-1999. Instituto Nacional de Cancerología, International Agency for Research on Cancer, Ministerio de la Protección Social; 2005.

7. Instituto Nacional de Cancerología. Grupo de Investigación clínica. Guía de práctica clínica explícita basada en la evidencia sobre la eficacia de la vacuna contra Streptococcus pneumoniae y virus Influenza en pacientes oncológicos. Bogotá: Instituto Nacional de Cancerología; 2006.

8. Christenson B, Lundbergh P, Hedlund J, Ortqvist A. Effects of a large scale intervention with influenza and 23-valent pneumococcal vaccines in adults aged 65 years or older: a prospective study. Lancet. 2001; 357: 1008-1011.

9. Moore A, Wiffen P, Lipsky B. Are the pneumococcal polysaccharide vaccine effective? Meta-analysis of the prospective trials. BMC Medicine. 2000 [internet]. Disponible en: http://biomedcentral.com/1471-2296/1/1 Consultado Enero de 2006.

10. Addiego JE, Ammann AJ, Schiffman G, Baehner R, Higgins G Hammond D. Response to Pneumococcal Polysaccharide Vaccine In Patients With Untreated Hodgkin's Disease: Children's Cancer Study Group Report. Lancet. 1980; 316(8192): 450453.

11. National Guideline Clearinghouse, Adult preventive health care: immunization. Norteamerica: Healthcare Infection Control Practices Advisory Committee; 2002 [internet]. Disponible en: http: / www.guideline.gov. Actualizado agosto 2002; Consultado Julio de 2006.

12. National Guideline Clearinghouse, Preventing pneumococcal disease among infants and young children. Norteamerica: Healthcare Infection Control Practices 
Advisory Committee; 2003 [internet]. Disponible en: http:/ www.guideline.gov. Actualizado septiembre 2003; Consultado Julio de 2005.

13. Congress of The United States-Office of Technology Assessment. (1978), Assessing the efficacy and safety of Medical Technologies. Washington, U.S. Government Printing Office [internet]. Disponible en: http://www.wws.princeton. edu/ota/ ns20/alpha_f.html. Consultado Noviembre de 2006.

14. Lázaro P. Evaluación de Tecnología Médica, Papeles de Gestión Sanitaria. Monografía no. II-94, Valencia, M/C/QEdiciones; 1994.

15. Castro R, Mokate KM. Evaluación económica y social de proyectos de inversión. Bogotá: Ediciones Uniandes, Alfaomega; 2004.

16. Instituto Nacional de Cancerología. Anuario Estadístico 2004. Bogotá: Instituto Nacional de Cancerología; 2005.

17. Sankaranarayanan R, Black RJ, Swaminathan R, Parkin DM. An overview of cancer survival in developing countries. In: Parkin DM. Cancer survival in developing countries. Lyon: International Agency for Research on Cancer, IARC Scientific Publications. No. 145; 1998.p. 135-173.

18. Globocan 2002. [Internet]. Disponible en: http://www-dep.iarc.fr/globocan/ database.htm Consultado em Agosto 2006. 